\title{
Performance evaluation of OFDM and SC-QAM backhaul provision on FTTH optical access networks including multi-core fiber riser
}

Maria Morant, Julián Bruno, Vicenç Almenar, Roberto Llorente

Maria Morant, Julián S. Bruno, Vicenç Almenar, Roberto Llorente, "Performance evaluation of OFDM and SC-QAM backhaul provision on FTTH optical access networks including multi-core fiber riser," Proc. SPIE 10945, Broadband Access Communication Technologies XIII, 1094509 (1 February 2019); doi: 10.1117/12.2509678

SPIE. Event: SPIE OPTO, 2019, San Francisco, California, United States 


\title{
Performance Evaluation of OFDM and SC-QAM Backhaul Provision on FTTH Optical Access Networks including Multicore Fiber Riser Maria Morant ${ }^{\mathrm{a}^{*}}$, Julián S. Bruno ${ }^{\mathrm{b}-\mathrm{c}}$, Vicenç Almenar $^{\mathrm{b}}$ and Roberto Llorente ${ }^{\mathrm{a}}$ \\ ${ }^{a}$ Nanophotonics Technology Centre, Universitat Politècnica de València (UPV), Valencia, Spain. \\ ${ }^{\mathrm{b}}$ Instituto de Telecomunicaciones y Aplicaciones Multimedia (ITEAM), UPV, Spain. \\ ${ }^{c}$ Laboratorio Procesamiento Digital, Universidad Tecnológica Nacional, Buenos Aires, Argentina. mmorant@ntc.upv.es
}

\begin{abstract}
This paper proposes and evaluates experimentally the performance of single-carrier QAM (SC-QAM) and OFDM-QAM signals for next-generation backhaul over a deep fiber-to-the-home (FTTH) network comprising up to $50 \mathrm{~km} \mathrm{SSMF}$ combined with in-building transmission over $150 \mathrm{~m}$ of 4-core multi-core fiber in order to reach the cellular transmission equipment usually located in the roof. The data signals are generated with commercial off-the-shelf (COTS) components using QAM modulation orders up to 256QAM. OFDM and SC-QAM transmission after 10-km SSMF PON and 150-m MCF in-building riser employing 256QAM provides $14.22-14.36 \mathrm{~Gb} / \mathrm{s}$ per channel. $50-\mathrm{km}$ PON is reached employing 128QAM signals. Channel aggregation is also investigated to increase the system capacity. The experimental results point out that aggregation of a second data channel is feasible employing the same components with a 3-dB received power penalty. The minimum received optical power level is evaluated experimentally for each signal. This approach enables operators to select the optimum modulation order depending on the distance and the received power level, providing up to $28.44 \mathrm{~Gb} / \mathrm{s}$ per user with two 256QAM channels.
\end{abstract}

Keywords: Multicore fiber, space division multiplexing, single-carrier QAM, OFDM, channel aggregation

\section{INTRODUCTION}

Next-generation fiber-to-the-home (FTTH) optical access networks target to provide high-bitrate services to a large number of users in an increasingly larger coverage area [1]. Moreover, the European Union aims to foster competition of incumbent and emerging operators by enabling actual sharing of the on-the-field optical infrastructure, as indicated by latest telecom directives [2]. In order to satisfy these requirements, this paper proposes the use of multicore fiber (MCF) in the in-building network, including the optical riser which connects the on-the-street FTTH fiber to the in-premises optical network terminal (ONT) through the building [3]. The riser fiber is commonly implemented by a thick and heavy multi-fiber cable with up to 288 single-mode fibers [4], each one connecting an individual home. Some buildings already have multimode fibers installed for local area network applications [1],[3]. However, multimode and plastic fiber networks usually require 850 or $1300 \mathrm{~nm}$ wavelength transmission [3], which differs from the $1550 \mathrm{~nm}$ transmission employed in the access network, thus requiring a residential gateway interface between the FTTH and the in-building network [1]. Moreover, the requirements may differ for each operator or subscribed service, which makes the in-building dimensioning even more difficult. The proposed in-building network can be used to replace the current multi-fiber cable riser with MCF media, which enables the support of different operators, from different locations if needed, as depicted in Figure 1. This implementation has the advantages of reduced weight and increased flexibility without the necessity of gateways and is especially interesting for large residential and business buildings, as found in major European cities.

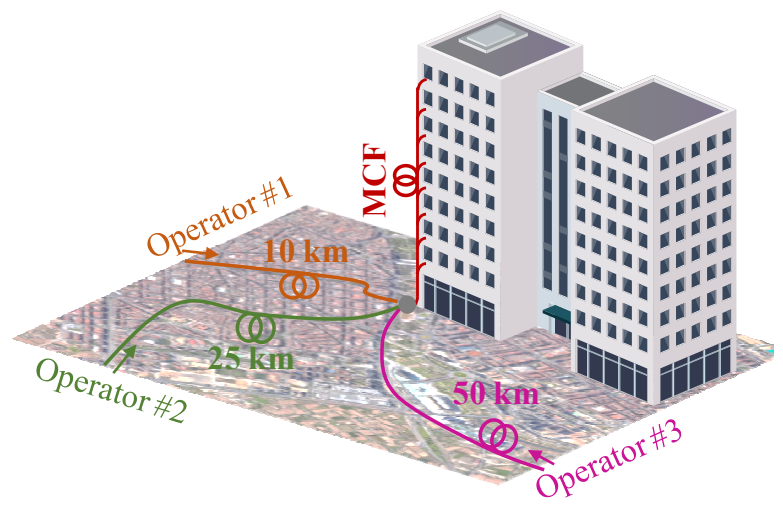

Figure 1. Application scenario for multi-operator PON transmission with MCF in-building network 
To date, multi-carrier orthogonal frequency division multiplexing (OFDM) and single-carrier (SC) signals employing quadrature amplitude modulation (QAM) are the modulations of choice for next-generation PON access, as defined by FSAN [5], and for latest hybrid fiber-coax (HFC) networks supporting DOCSIS 3.1 standard. OFDM enables the adaptive tune of the modulation format and power per carrier to optimize the transmission path [1], while single-carrier signals enables a simpler implementation [6].

In this paper, we evaluate experimentally the optical performance of OFDM and SC-QAM signals after the radio-overfiber transmission over 10, 25 and $50 \mathrm{~km}$ passive optical networks (PONs) and $150 \mathrm{~m}$ MCF in-building network. The data signals are generated with commercial off-the-shelf (COTS) components using QAM modulation orders ranging from 16 to 256QAM. The experimental analysis evaluates the minimum received power level required at user premises for free-error transmission. With this information, the operators are able to select from the central office the optimum modulation order depending on the distance to user premises and the received power level, maximizing the provided user capacity. In order to increase the user capacity, channel aggregation is also evaluated experimentally using the same transmission components.

\section{EXPERIMENTAL EVALUATION}

\subsection{Experimental setup and signal generation}

Figure 2 shows the experimental setup employed for the performance comparison of SC and OFDM signals after PON and MCF in-building transmission. The electrical signals are generated using an FPGA from Xilinx (Virtex-7 XC7VX485T-2) with a digital-to-analog converter (DAC) operating at $4 \mathrm{GS} / \mathrm{s}$ (Euvis DAC MD657B) to show experimentally that is possible to reach a high bitrate with commercial off-the-shelf (COTS) components.

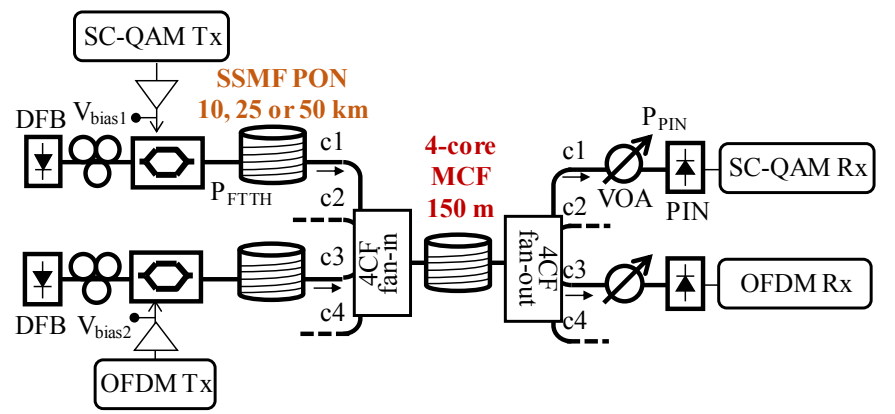

Figure 2. Experimental setup for SSMF PON and MCF in-building transmission of OFDM and SC-QAM signals

Table 1. Data signal generation parameters

\begin{tabular}{|c|c|c|c|c|c|c|c|c|c|c|}
\hline \multicolumn{5}{|c|}{ Single-carrier QAM } & \multicolumn{6}{|c|}{ OFDM } \\
\hline \multirow{2}{*}{\multicolumn{2}{|c|}{ Roll-off $=0.1$}} & & \multirow{2}{*}{\multicolumn{2}{|c|}{ Samples per symbol $=2.25$}} & \multirow{2}{*}{\multicolumn{3}{|c|}{$\begin{array}{c}\text { Subcarriers }=512 \\
\text { Active subcarriers }=467\end{array}$}} & \multirow{2}{*}{\multicolumn{3}{|c|}{$\begin{array}{c}\text { Subcarrier sep. }=3.91 \mathrm{MHz} \\
\text { Cyclic prefix }=16\end{array}$}} \\
\hline & & & & & & & & & & \\
\hline Modulation & 16QAM & 64QAM & 128QAM & 256QAM & Modulation & 16QAM & & $\mathrm{AM}$ & 128QAM & 256QAM \\
\hline Data-rate & $7.11 \mathrm{~Gb} / \mathrm{s}$ & $10.66 \mathrm{~Gb} / \mathrm{s}$ & $12.44 \mathrm{~Gb} / \mathrm{s}$ & $14.22 \mathrm{~Gb} / \mathrm{s}$ & Data-rate & $7.18 \mathrm{~Gb} / \mathrm{s}$ & 10. & $7 \mathrm{~Gb} / \mathrm{s}$ & $12.57 \mathrm{~Gb} / \mathrm{s}$ & $14.36 \mathrm{~Gb} / \mathrm{s}$ \\
\hline
\end{tabular}

The main parameters employed for the signal generation and the provided data-rate using 16 to 256QAM modulation are summarized in Table 1.

The SC-QAM signals are generated with a root raised cosine pulse shape using a roll-off factor of 0.1. For an efficient use of the DAC spectrum, the single-carrier in-phase and quadrature branches are generated with 2.25 samples per symbol employing a polyphase filter structure and then digitally upconverted to $1 \mathrm{GHz}$ (center of the transmission band).

The baseband OFDM signal is generated using a 1024-point fast Fourier transform (FFT) but, due to the Hermitian symmetry in frequency to generate a real valued signal, only 512 subcarriers are available for data transmission. Due to analog filtering and in order to avoid interference with the DAC clock, 45 subcarriers are set null (16 subcarriers located at low frequency, 24 at high frequency and 5 around $2 \mathrm{GHz}$ ). The cyclic prefix length is set to 16 samples to cope with the impulse response length of the filters and the chromatic dispersion of long-reach optical links. More information about the OFDM generation can be found in [7]. 
These electrical signals are modulated with Mach-Zehnders over two distributed feedback laser (DFB) operating at the same wavelength $(1555.75 \mathrm{~nm})$ to evaluate the worst-case optical crosstalk scenario. Different PON links of 10, 25 and $50 \mathrm{~km}$ of standard single mode fiber (SSMF) are evaluated maintaining the optical power level at the input of the PON link at $\mathrm{P}_{\mathrm{FTTH}}=6.6 \mathrm{dBm}$ without optical amplification.

As it was depicted before, with the proposed approach, no gateway interface is needed between the SSMF and the MCF media proposed for in-building transmission. The signals after PON transmission are directly injected in one of the cores of a 4-core single-mode fibre $(4 \mathrm{CF})$ without detection or remodulation employing 3D fan-in/fan-outs. In this experimental evaluation, the different PON signals are injected into cores 1 and 3 (which have the lowest core pitch $\Lambda \mathrm{c}$ ) in order to evaluate the system performance under the worst inter-core crosstalk conditions. In this demonstration, the 4CF is rolled up in a spool with 45-cm diameter, as the higher the bending radius, the higher the inter-core crosstalk [8].

At the receiver, variable optical attenuators (VOAs) are used to evaluate different optical power levels arriving to the photodiode $\left(\mathrm{P}_{\mathrm{PIN}}\right)$ with the same injected power level into the fiber. Direct PIN photodetection is implemented before sampling the signal with an oscilloscope (DSO 91304A) for MATLAB post-processing. Integrated reception can be performed also in real-time using an FPGA device and an EV10AQ190A analog-to-digital converter (ADC) [7].

For demodulation, both signals use a preamble for synchronization and channel estimation. OFDM includes a 1-weight channel equalizer per subcarrier while SC-QAM signals are downconverted to baseband and processed with a 40 taps linear equalizer to cancel inter-symbol interference. Once equalized, the error vector magnitude (EVM) is obtained and the bit error rate (BER) is calculated [9].

\subsection{Single-channel performance analysis}

Figure 3 shows the BER performance of both SC-QAM (filled symbols) and OFDM signals (open symbols) after 10, 25 and $50 \mathrm{~km}$ SSMF PON and $150 \mathrm{~m}$ MCF transmission. In the results depicted in Figure 3, the dashed horizontal line represents the BER threshold at $3.8 \cdot 10^{-3}$ needed to obtain error free transmission with $7 \%$ redundancy hard-decision forward error correction (HD-FEC) [10].
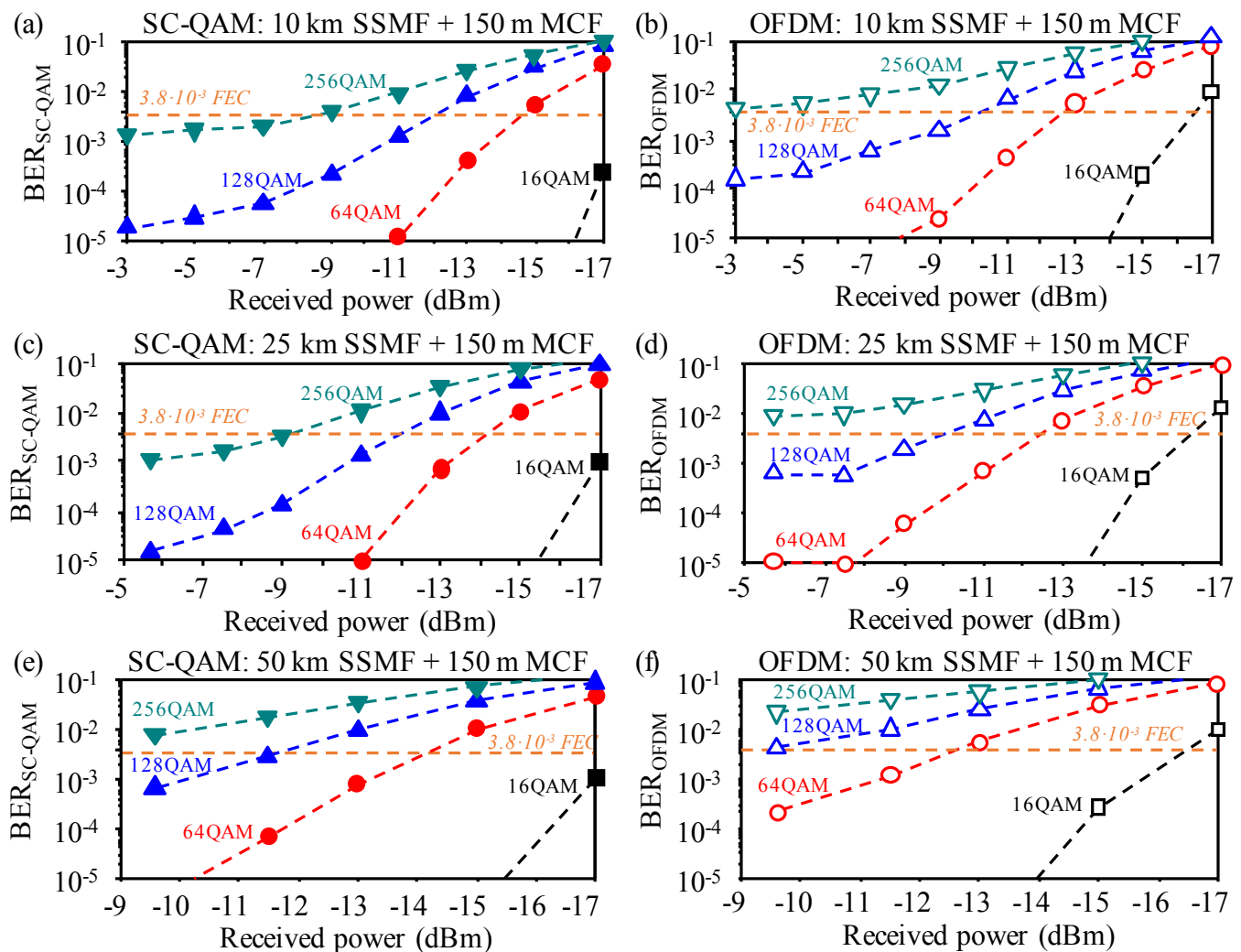

Figure 3. BER performance of single-carrier (left) and OFDM (right) after: (a)(b) $10 \mathrm{~km}$, (c)(d) $25 \mathrm{~km}$ and (e)(f) $50 \mathrm{~km}$ SSMF PON and $150 \mathrm{~m}$ MCF transmission vs. received optical power level 
For a closer comparison, in Figure 4 the BER performance of both SC-QAM and OFDM signals is reported for a $25 \mathrm{~km}$ SSMF PON link including $150 \mathrm{~m}$ MCF in-building transmission. Looking at the results depicted in Figure 3 to Figure 5, it can be observed that the performance of the SC-QAM signals is better than using OFDM-QAM signals. This is due to the fact that SC-QAM signals have a much lower peak to average power ratio (PAPR) compared with OFDM signals. For this reason, the OFDM signals cannot be amplified as much as the SC-QAM signals in order to avoid nonlinear distortions caused by the electrical amplifiers and the electro-optical modulator. This behavior is confirmed in Figure 5 constellations for a received power level of $\mathrm{P}_{\mathrm{PIN}}=-5.7 \mathrm{dBm}$.

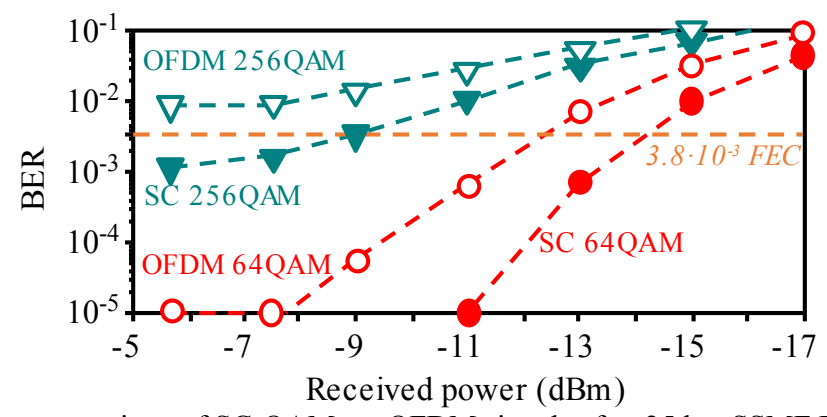

Figure 4. Performance comparison of SC-QAM vs. OFDM signals after $25 \mathrm{~km}$ SSMF PON and $150 \mathrm{~m} \mathrm{MCF}$ radio-over-fiber transmission

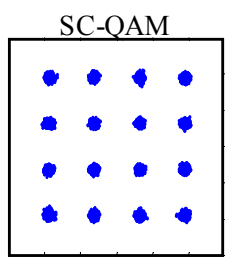

(a)
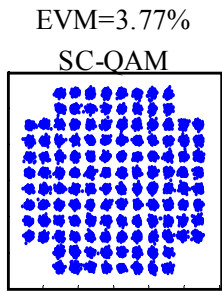

(c) $\mathrm{EVM}=3.88 \%$

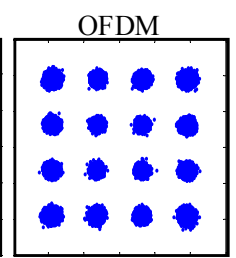

$\mathrm{EVM}=5.2 \%$ OFDM

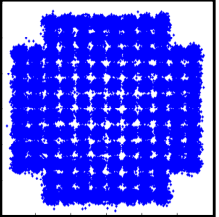

$\mathrm{EVM}=5.09 \%$

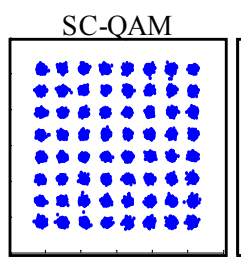

(b)

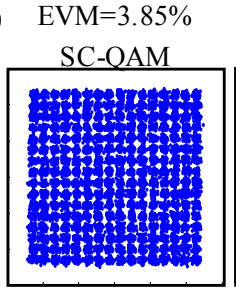

(d)

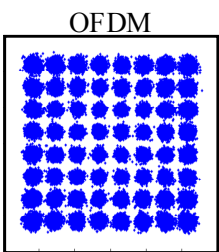

$\mathrm{EVM}=5.19 \%$

OFDM

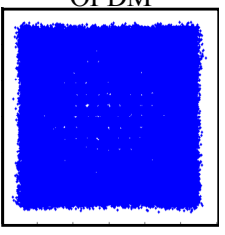

$\mathrm{EVM}=5.21 \%$

Figure 5. Received constellations and measured EVM of SC and OFDM signals after $25 \mathrm{~km} \mathrm{SSMF}$ and $150 \mathrm{~m}$ MCF transmission for $\mathrm{P}_{\mathrm{PIN}}=-5.7 \mathrm{dBm}$ employing (a) 16QAM, (b) 64QAM, (c) 128QAM and (d) 256QAM

Regarding the optical reach, as it can be extracted from Figure 3(e)(f), a $50 \mathrm{~km} \mathrm{SSMF} \mathrm{PON} \mathrm{transmission} \mathrm{followed} \mathrm{by}$ $150 \mathrm{~m}$ MCF meets the desired 3.8 $10^{-3}$ BER threshold with 128QAM for both signals. However, OFDM-QAM signals require a $+2.5 \mathrm{~dB}$ higher received power level, $\mathrm{P}_{\mathrm{PIN}}$ 50km OFDM-128QAM $\geq-9 \mathrm{dBm}$, compared with SC-QAM signals which only need $\mathrm{P}_{\mathrm{PIN}} 50 \mathrm{~km} \mathrm{SC}-128 \mathrm{QAM} \geq-11.5 \mathrm{dBm}$.

\subsection{Channel aggregation performance}

In order to further increase the user capacity, channel aggregation is evaluated with the generation of a second OFDM/SC-QAM channel with the same FPGA and a second DAC module following the schematic depicted in Figure 6(a). This second DAC module -labelled DAC2 in Figure 6(a)- is also an Euvis MD657B but it is configured to operate in return zero mode, which gives a flatter DAC frequency response in the second Nyquist band. With this approach, two copies of the signal are generated: one between 0 and $2 \mathrm{GHz}$ and another between 2 to $4 \mathrm{GHz}$. The signal obtained at 2-4 GHz is band-pass filtered (BPF) to obtain the second data channel without employing any mixer for upconversion. The new data channel is aggregated to the data channel generated with the first DAC module, located between 0 and $2 \mathrm{GHz}$. 


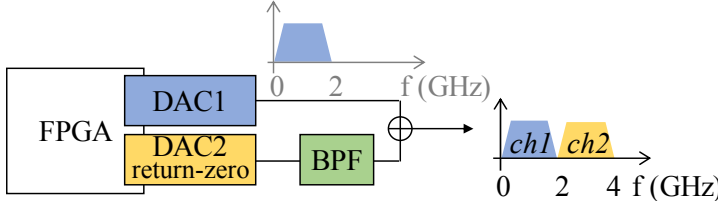

(a)

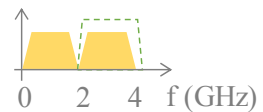

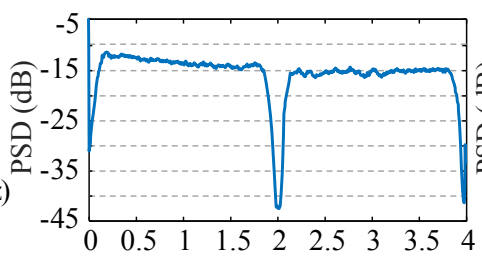

(b)

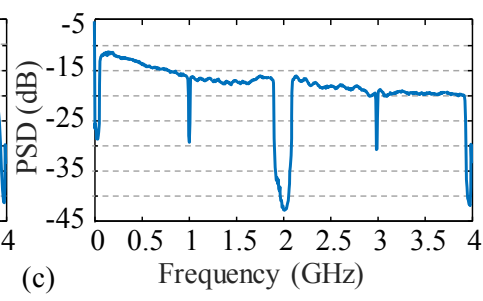

(c)

Figure 6. (a) Block diagram for channel aggregation employing an FPGA and two DAC modules. Measured spectrum for (b) two SC-QAM channels and (c) two OFDM-QAM channels received after $10 \mathrm{~km}$ SSMF PON and $150 \mathrm{~m}$ MCF with a received power level of $\mathrm{P}_{\mathrm{PIN}}=-7 \mathrm{dBm}$

Figure 6(b)-(c) show the resulting spectrum of both aggregated channels after $10 \mathrm{~km} \mathrm{SSMF} \mathrm{PON} \mathrm{and} 150 \mathrm{~m}$ MCF transmission. As it was commented before, it can be observed that the power spectral density (PSD) of the OFDM signals is lower than for SC-QAM signals. This difference is even more significant for the second channel with higher frequency components, as represented in Figure 6(b). For this reason, when channel aggregation is implemented, the limiting factor is the performance of the second channel. As it is reported in the received constellations included Figure 7, we confirmed experimentally that the second data channel requires $+3 \mathrm{~dB}$ extra received power compared with the first data channel, while doubling the system capacity for a $10 \mathrm{~km}$ PON transmission followed by $150 \mathrm{~m}$ of MCF.

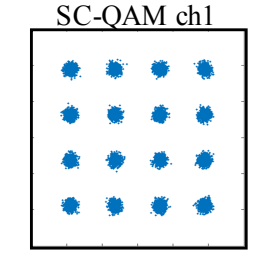

(a) $\mathrm{EVM}=5.64 \%$

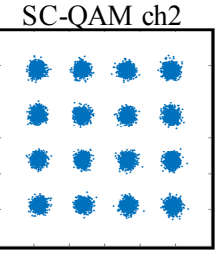

$\mathrm{EVM}=7.57 \%$

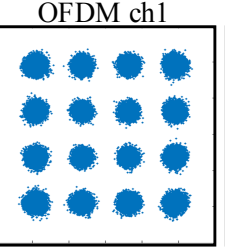

(b) $\mathrm{EVM}=7.41 \%$
OFDM ch2

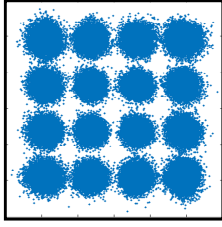

$\mathrm{EVM}=12.4 \%$

Figure 7. (a) Received SC-QAM and (b) OFDM constellations for aggregated ch1 and ch2 with 16QAM after $10 \mathrm{~km}$ SSMF $\mathrm{PON}$ and $150 \mathrm{~m} \mathrm{MCF}$ in building transmission for $\mathrm{P}_{\mathrm{PIN}}=-7 \mathrm{dBm}$

Considering the received power requirements obtained for that optical link in Figure 3(a)(d), the optimum modulation format to provide the maximum data rate with error-free transmission is represented in Figure 8 . This capacity evaluation is also valid for 25 and $50 \mathrm{~km}$ PONs, except for the case of 256QAM that is only met for SC-QAM signals. With the proposed approach, operators can switch from SC-QAM to OFDM and select the optimum modulation order (in this case from 16 to 256QAM) depending on the received power level to maximize the data-rate provided to the user.

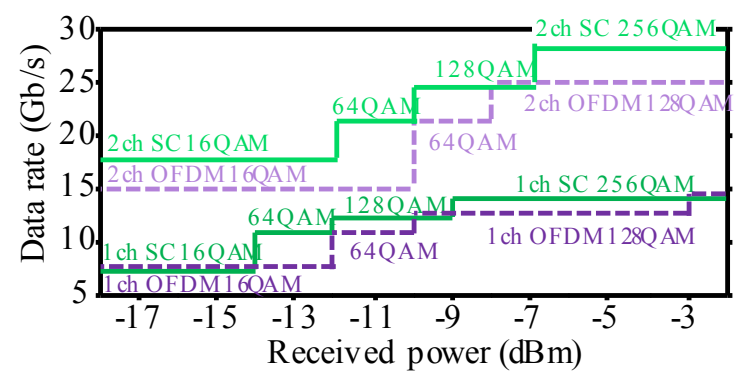

Figure 8. Provided data rate with channel aggregation of OFDM and SC-QAM after $10 \mathrm{~km}$ SSMF PON and $150 \mathrm{~m}$ MCF in-building transmission vs. received power level $\mathrm{P}_{\mathrm{PIN}}$

\section{CONCLUSION}

This paper proposes and evaluates experimentally the performance of single-carrier QAM and OFDM-QAM signals for multiple radio-over-fiber transmission over deep FTTH networks and in-building transmission over $150 \mathrm{~m}$ of 4-core MCF. In-building MCF media is proposed to replace the riser fiber currently implemented with multi-fiber cable. This proposal enables the signal provision from different operators, that could be at the same or at different distances from the final user. In this experimental work, deep FTTH transmission through 10, 25 and $50 \mathrm{~km}$ SSMF PONs are evaluated for this purpose. 
Data rates of 14.22 and $14.36 \mathrm{~Gb} / \mathrm{s}$ are provided with a single SC-QAM and OFDM channel, respectively, after 10-km SSMF PON and 150-m MCF in-building riser employing 256QAM modulation. The FTTH transmission can reach up to $50 \mathrm{~km}$ PON employing 128QAM modulation, thus providing $12.44 \mathrm{~Gb} / \mathrm{s}$ per channel with SC-QAM signals or 12.57 $\mathrm{Gb} / \mathrm{s}$ per channel with OFDM-QAM signals. Channel aggregation is also evaluated employing the same devices but using a second DAC in return-zero configuration. With the transmission of a second channel, the capacity is doubled with the requirement of extra $+3 \mathrm{~dB}$ received optical power. The proposed approach enables the operators to select the optimum modulation order depending on the distance to user premises and the received power level, providing up to $28.44 \mathrm{~Gb} / \mathrm{s}$ with two 256QAM channels.

\section{ACKNOWLEDGEMENTS}

This research was supported in part by Spain National Plan MINECO FEDER UE TEC2015-70858-C2-1-R XCORE and TEC2015-70858-C2-2-R OPTODSP projects, both with FEDER funds. M. Morant work supported by Spain Juan de la Cierva IJCI- 2016-27578 grant. Project HYPERCONN grant Leonardo 2018 by Fundacion BBVA is also acknowledged.

\section{REFERENCES}

[1] Koonen A.M.J. and Tangdiongga, E., "Photonic home area networks," IEEE/OSA Journal in Lightwave Technology 32(4), 591-604 (2014).

[2] European Parliamentary Research Service, "The new European electronic communications code," EU Legislation in Progress (2017).

[3] Keiser, G., et al., "Practical aspects of access network indoor extensions using multimode glass and plastic optical fibers," Fiber and Integrated Optics 31(4), 221-228 (2012).

[4] TE connectivity, Fiber optic indoor/outdoor cables, 114-92002, Rev G (2013).

[5] Huawei Technology, Next-Generation PON Evolution, (2010).

[6] Buzzi, S., D’Andrea, C., Foggi, T., Ugolini, A. and Colavolpe, G., "Single-carrier modulation versus OFDM for millimeter-wave wireless MIMO," IEEE Transactions on Communications, vol. 66, no. 3, pp. 1335-1348 (2018).

[7] Bruno, J. S., Almenar, V., Valls, J. and Corral, J. L., "Real-time 20.37 Gb/s optical OFDM receiver for PON IM/DD systems," OSA Optics Express 26(15), 18817-18831 (2018).

[8] Morant, M., Macho, A. and Llorente, R., "On the suitability of multicore fiber for LTE-Advanced MIMO optical fronthaul systems," IEEE/OSA Journal in Lightwave Technology 34(2), 676-682 (2016).

[9] Schmogrow, R., et al., "Error vector magnitude as a performance measure for advanced modulation formats," IEEE Photonics Technology Letters 24(1), 61-63 (2012).

[10] "Forward error correction for high bit-rate DWDM submarine systems," ITU-T Recommendation Std. G.975.1 (2004). 